PHYSICAL REVIEW D 92, 069903(E) (2015)

\title{
Erratum: Alternative formation model for antideuterons from dark matter [Phys. Rev. D 91, 123536 (2015)]
}

\author{
L. A. Dal and A. R. Raklev \\ (Received 11 September 2015; published 22 September 2015; publisher error corrected 29 September 2015) \\ DOI: 10.1103/PhysRevD.92.069903 \\ PACS numbers: 95.35.+d, 12.60.Jv, 27.10.+h, 98.70.Sa, 99.10.Cd
}

In proofreading the paper, the absence of several important references to the Monte Carlo event generators used was missed. This is unfortunate both in terms of later reproducibility, and for the lack of acknowledgment of the large body of work these generators represent. In the first paragraph of Sec. IV the paper should acknowledge the use of HERWIG++ 2.6.0 with Refs. [1,2] and PYTHIA 8.186 with Refs. [3,4]. At the beginning of the third paragraph of Sec. IV A PHOJET should be referenced with $[5,6]$.

Naturally, the conclusions of the paper remain unchanged.

[1] M. Bahr et al., Herwig + + physics and manual, Eur. Phys. J. C 58, 639 (2008).

[2] K. Arnold et al., Herwig ++2.6 release note, arXiv:1205.4902.

[3] T. Sjostrand, S. Mrenna, and P.Z. Skands, PYTHIA 6.4 physics and manual, J. High Energy Phys. 05 (2006) 026.

[4] T. Sjostrand, S. Mrenna, and P. Z. Skands, A brief introduction to PYTHIA 8.1, Comput. Phys. Commun. 178, 852 (2008).

[5] R. Engel, Photoproduction within the two component dual parton model. 1. Amplitudes and cross-sections, Z. Phys. C 66, 203 (1995).

[6] R. Engel and J. Ranft, Hadronic photon-photon interactions at high-energies, Phys. Rev. D 54, 4244 (1996). 